ESJ Social Sciences

\title{
Reseaux de solidarite et developpement du secteur informel feminin en cote d'ivoire : etude de cas des femmes entrepreneures a bouaké
}

\author{
Valoua Fofana \\ Adou Paul Venance \\ Université Alassane Ouattara, Côte d'Ivoire \\ Camara Mariam \\ Centre Ivoirien de Recherches Economiques et Sociales (CIRES), \\ Côte d'Ivoire
}

\section{Doi:10.19044/esj.2021.v17n5p178}

Submitted: 28 December 2020

Accepted: 08 February 2021

Published: 28 February 2021
Copyright 2021 Author(s)

Under Creative Commons BY-NC-ND

4.0 OPEN ACCESS

Cite As:

Fofana V., Venance P.A. \& Mariam C. (2021). Reseaux de solidarite et developpement du secteur informel feminin en cote d'ivoire : etude de cas des femmes entrepreneures a bouaké. European Scientific Journal, ESJ, 17(5), 178. https://doi.org/10.19044/esj.2021.v17n5p178

\section{Résumé}

Les femmes développent de plus en plus de stratégies entrepreneuriales pouvant faciliter le développement et la pérennité de leurs petites unités économiques. En dépit des niveaux très faibles du capital humain, matériel et financier, elles parviennent à devenir d'importantes entrepreneures par des stratégies de mutualisation tout aussi innovantes qu'hybrides. Ces systèmes de solidarité font l'objet de cet article. Il vise, notamment, à comprendre et analyser le processus de transformation de ces activités informelles en lien avec les différentes formes de solidarités auxquelles participent les promotrices. Pour ce faire, une enquête mixte (qualitative et quantitative) a été conduite auprès d'un échantillon aléatoire simple de 96 femmes revendiquant une réussite économique dans leurs activités à Bouaké. A terme, l'analyse des données empiriques au moyen de la démarche interactionniste suggère : (i) les déterminants des choix des réseaux par les femmes; (ii) l'accompagnement des activités par ces réseaux; (iii) la sécurisation de celles-ci par la pérennisation des activités et ; (iv) les tensions inhérentes aux relations interpersonnelles au sein des réseaux de solidarité. 
Mots clés : Réseaux sociaux, solidarité, développement, secteur informel, femmes

\title{
Solidarity Networks and Development of the Informal Women Sector in Cote d'Ivoire: Case Study of Women Entrepreneurs in Bouake
}

\author{
Valoua Fofana \\ Adou Paul Venance \\ Université Alassane Ouattara, Côte d'Ivoire \\ Camara Mariam \\ Centre Ivoirien de Recherches Economiques et Sociales (CIRES), \\ Côte d'Ivoire
}

\begin{abstract}
Women are developing more and more entrepreneurial strategies that can facilitate the development and sustainability of their small economic units. Despite the very low levels of human, material and financial capital, they manage to become important entrepreneurs through mutualization strategies that are as innovative as they are hybrids. These solidarity systems are the subject of this paper. It aims, in particular, to understand and analyze the transformation process of these informal activities in connection with the different forms of solidarity in which the promoters participate. To do this, a mixed survey (qualitative and quantitative) was conducted with a simple random sample of 96 women claiming economic success in their activities in Bouaké. Ultimately, the analysis of empirical data using the interactionist approach suggests: (i) the determinants of network choices by women; (ii) the support of activities by these networks; (iii) securing them through the sustainability of activities and; (iv) the tensions inherent in interpersonal relationships within solidarity networks.
\end{abstract}

Keywords: Social networks, solidarity, development, informal sector, women

\section{Introduction}

En plus d'être plus nombreuses dans l'économie informelle, comparativement aux hommes (OIT, 2019) ${ }^{1}$, les femmes exercent généralement les activités les plus vulnérables et peu productives. Les contraintes sociales, financières et institutionnelles annihilent la progression de leurs activités au point de les limiter au seul rôle de pourvoyeuses de

${ }^{1}$ En Afrique, 89,7 pour cent des femmes occupent un emploi informel, contre 82,7 pour cent d'hommes. 
moyens de subsistance des ménages. Elles ont une certaine prédilection pour les activités commerciales et les services, plus faciles à créer et demandant peu de qualifications spécifiques (Ba Gning, 2013). Du coup, se pose constamment la question de l'autonomisation des femmes exerçant dans l'économie informelle. Leurs marges de manœuvres sont très limitées au point où elles se heurtent très souvent à un certain nombre de contraintes structurelles et sociales lorsqu'elles aspirent à dynamiser leurs activités. Au niveau structurel par exemple, les femmes, dans leurs rapports avec les fournisseurs et les banquiers, sont confrontées à un déficit de crédibilité ; quant au niveau social, elles font face aux stéréotypes et préjugés (Fofana et al, 2020). Nonobstant ces contraintes, l'autonomisation des femmes dans l'économie informelle a quelque peu progressé ces dernières années. Certaines de leurs activités sont de plus en plus modernes et dynamiques passant d'activités domestiques à des affaires florissantes capables de créer des revenus substantiels et des emplois. Certaines y parviennent en courtisant les banques de proximité ou agences de microfinances (Fofana, 2017) en échange d'hypothèques (très souvent) quand d'autres innovent des formes de solidarités collectives (OIT, 2018).

Très peu d'études ont mis en exergue l'impact de ces formes de solidarité collectives ou réseaux sociaux sur les activités informelles féminines. Ba Gning (2013) trouve que les stratégies d'accumulation entraînent la recherche d'alliances de type familial, syndical, politique ou religieux. C'est pourquoi, les opératrices informelles s'insèrent dans des organisations en mesure de leur permettre d'obtenir des facilités d'acquisition et de distribution des marchandises dans le marché intérieur et à l'étranger. Cette idée est également présente dans les travaux empiriques de Plateau (2000) puis de ceux de Hoff et Sen (2006) qui reconnaissent en partie que les liens familiaux et de parenté peuvent être moteurs dans la mise en place de contrats d'assistance mutuelle dans un contexte d'imperfections de marché. Di Falco et Bulte (2010) ne manquent toutefois pas de souligner l'impact négatif de cette forme de solidarité sur la rentabilité des activités concernées lorsqu'ils révèlent l'existence de normes distributives donnant lieu à des comportements de passager clandestin et désincitant les individus à s'auto-assurer contre les chocs. Face à ces normes distributives qui desservent leurs activités, certaines entrepreneures informelles innovent. Elles participent à des tontines pour protéger leurs économies des demandes de consommation émanant de leurs maris (Anderson et Baland, 2002). De par leur position moins privilégiée, les femmes trop pauvres pour épargner trouvent sécurité et protection sociale dans les initiatives collectives fondées sur des systèmes de dons et des contredons obligatoires d'une part; de recours à des emprunts en cas de difficulté économique d'autre part (Rossetti, 2015). Dans la littérature existante, des travaux empiriques ont pu révéler néanmoins que ces systèmes d'emprunts ou de dons et contredons ne présentent pas que des avantages. Il arrive que ces 
femmes se retrouvent dans des situations décrites par Baland et al. (2007). S'intéressant aux comportements d'emprunt des individus, ces auteurs ont constaté, en effet, que certaines personnes empruntent de l'argent bien qu'elles n'aient aucune contrainte de liquidité, et ce simplement pour signaler à leurs proches qu'ils sont dans l'incapacité de leur fournir une assistance financière. Une approche incluant les conflits permet, par ailleurs, de ne pas entrer dans une vision idéalisée et angélique d'une solidarité dite africaine marquant les relations sociales d'autant que les inégalités et les tensions peuvent caractériser les acteurs sociaux entrant en relation (Mbueselir, 2010).

Quoique, fonctionnant grâce aux réseaux de solidarité, l'économie informelle ne puisse pas faire fi des rivalités et des conflits inhérents aux relations sociales, les risques et les chocs omniprésents en font un terrain favorable au développement de nouvelles formes de médiation sociale et de solidarité citadine face à l'affaiblissement des institutions sociales traditionnelles en proie à l'urbanisation et à la crise économique (Marie, Vuarin, Leimdorfer et al., 2008). La présente contribution s'inscrit dans cette perspective et repose sur une étude appliquée aux microentrepreneures du secteur informel féminin de Bouaké. La survie des activités de la plupart des femmes exerçant dans l'informel à Bouaké passe par les réseaux sociaux dont la cohésion repose sur des mécanismes de la réciprocité et de la redistribution. Réunies en associations ou coopératives selon les affinités identitaires, confessionnelles ou professionnelles, elles développement des liens de solidarité afin de faire face aux faiblesses qui affectent leurs activités économiques et assurer par ailleurs leur équilibre économique et sociale. Quelles formes de réseaux sociaux mobilisent les micro-entrepreneures de Bouaké? Comment ces réseaux sociaux leur permettent-ils de développer leurs activités ? L'objectif général poursuivi dans cette étude est de comprendre les logiques qui sous-tendent les formes de réseaux sociaux mobilisés par les femmes dans l'exercice de leurs activités afin de déterminer dans quelle mesure ceux-ci leur permettent de développer leurs activités. L'hypothèse générale émise et que nous nous attelons à tester dans cet article est : le recours aux réseaux sociaux comme forme d'assurance informelle constitue pour les micro-entrepreneures une forme privilégiée de résilience face à la survenance de chocs dans la conduite de leurs activités, mieux un instrument privilégié de développement de celles-ci. La vérification de cette hypothèse obéit à une démarche scientifique dont la méthodologie.

\section{Méthodologie}

\subsection{Zone d'étude}

La collecte de données empiriques de cette étude a lieu à Bouaké. Capitale du pays Baoulé, la ville de Bouaké est le Chef-lieu de la région du Gbêkê, située au centre de la Côte d'Ivoire. Sa position de plate-forme 
économique et la densité de sa population ${ }^{2}$ ont favorisé une extension rapide de sa structure urbaine. A la faveur de cette urbanisation dynamique et des facteurs démographiques, la ville de Bouaké se compose de 39 quartiers y compris quelques villages alentour. Outre le grand marché, principal centre d'activité, certains quartiers ont leur propre centre commercial en raison de leur situation géographique par rapport au centre-ville et des coûts d'occupation des espaces commerciaux sur celui-ci situé en centre-ville. En tant que lieu de concentration d'activités économiques d'une part et d'implantations de groupements affinitaires et professionnels d'autre part, nous avons fait le focus sur certains de ces marchés secondaires pour la collecte de données auprès de certaines femmes exerçant dans le commerce. N'ayant pas d'espace dédié à leurs activités, les artisanes ont été approchées dans quatre quartiers (Commerce, Broukro, Air-France, Ahougnansou) dans leurs ateliers, salons de coiffure et de beauté, restaurants ou autres établissements spécialisés selon leur représentativité et leur appartenance à un réseau social.

\subsection{Population à l'étude et échantillon}

La population concernée par l'étude est essentiellement constituée de femmes exerçant des activités informelles et appartenant à des réseaux sociaux. Le choix des participantes à l'enquête relève de la technique d'échantillonnage par grappes d'abord pour la sélection du groupe d'appartenance puis l'échantillonnage aléatoire simple effectué dans la sélection des enquêtées. De manière concrète, une fois le groupe social identifié, la liste complète des membres de la population observée et l'information pour les contacter ont été fournies par la représentante légale, nous avons procédé à la détermination de la taille de l'échantillon. Pour ce faire, nous avons sélectionné pour chaque association ou groupement, un échantillon aléatoire simple reposant sur une base de sondage de $10 \%$. Cela signifie que chaque participante inscrite sur notre liste a eu 1 chance sur 10 d'être sélectionnée. La probabilité de sélection est la même pour chaque femme parmi les populations des différents réseaux quels que soient leur taille et le secteur d'activité de leurs membres. Au final, le tableau de répartition des enquêtées par réseau se présente dans le tableau ci-après :

\footnotetext{
${ }^{2}$ Selon les résultats officiels du Recensement Général de la Population et de l'Habitat (RGPH) fournis par l'Institut National de la Statistique (INS) de Côte d'Ivoire, la population urbaine de Bouaké est passée de 461617 habitants (1998) à 536189 habitants (2014), soit un taux d'accroissement moyen annuel de 4,66\% (RGPH 1998et 2014).
} 
Tableau $\mathbf{n}^{\circ} 1$ : Constitution de l'échantillon

\begin{tabular}{|l|c|c|c|}
\hline \multicolumn{1}{|c|}{ Réseau d'appartenance } & $\begin{array}{c}\text { Taille de la } \\
\text { population }\end{array}$ & $\begin{array}{c}\text { Taille de } \\
\text { l'échantillon }\end{array}$ & Fréquences. \\
\hline $\begin{array}{l}\text { Union des Femmes Entrepreneures de la } \\
\text { Région de Gbêkê (UFERG) }\end{array}$ & 230 & 23 & $23,96 \%$ \\
\hline $\begin{array}{l}\text { Union des femmes couturières de } \\
\text { Bouaké (UFCB) }\end{array}$ & 230 & 23 & $23,96 \%$ \\
\hline $\begin{array}{l}\text { Coopérative des Femmes du Secteur du } \\
\text { Vivrier de la Région du Gbêkê }\end{array}$ & 240 & 24 & $25 \%$ \\
\hline $\begin{array}{l}\text { Association Sœurs Unies } \\
\text { d'Ahougnassou }\end{array}$ & 260 & 26 & $27,08 \%$ \\
\hline \multicolumn{1}{|c|}{ TOTAL } & 960 & 96 & $100 \%$ \\
\hline
\end{tabular}

Sources : Données d'enquêtes, 2020

\subsection{Matériels}

Le contexte associatif des participantes et le souci de représentativité nous ont amené à procéder par une approche mixte dans la collecte de données empiriques. Nous avons ainsi fait appel à deux types d'outils (quantitatif et qualitatif) dans ce processus. Le questionnaire d'enquête invoqué pour le recueil de données quantitatives est composé de 3 axes thématiques portant sur (i) l'appartenance à un réseau, (ii) la création et développement de l'entreprise/activité, (iii) les caractéristiques sociodémographiques des participantes. L'outil de collecte de données utilisé est composé à la fois de questions fermées et de questions ouvertes nous permettant d'évaluer la motivation et la perception des participantes des mouvements associatifs. En outre, nous avons réalisé des entrevues individuelles semi-dirigées au moyen d'un guide d'entretien auprès des responsables (Présidente ou secrétaire) d'une part. D'une durée de 15 à 20 minutes, les entretiens ont tous été enregistrés (de façon numérique). Analysés subséquemment les entretiens ont permis de comprendre:

- Les motivations des entrepreneures à intégrer un réseau;

- Les modes d'articulation entre leurs activités professionnelles et leurs activités associatives;

- Les principales difficultés rencontrées.

D'autre part, des Groupes de discussion ont été organisés (2 discussions, compte tenu de la disponibilité des participantes) d'une durée moyenne de $60 \mathrm{mn}$ avec une dizaine de participantes par groupe.

\subsection{Traitement des données collectées}

Les analyses qualitatives ont été effectuées à l'aide du logiciel Lexica créé par «Le Sphinx Développement». Il permet de faire des études 
qualitatives ainsi que le traitement de données textuelles. Lexica permet d'analyser, non seulement, les textes de questions ouvertes, mais aussi, n'importe quel autre texte (Wanlin, 2007). L'une des possibilités offerte pour l'étude des textes est celle de l'analyse de contenu que nous avons également privilégiée dans cette étude. Inspirée d'une démarche parmi tant d'autres proposée par L'Écuyer (1990), l'analyse de contenu est une "méthode de classification ou de codification des divers éléments du matériel analysé, permettant à l'utilisateur d'en mieux connaître les caractéristiques et la signification ».

Concernant les analyses quantitatives, le logiciel sphinx a été utilisé afin de réaliser des statistiques descriptives et inférencielles. Sur ce logiciel, on a procédé à la tabulation des données et aux croisements des tableaux pour l'analyse multivariée.

\subsection{Méthode d'analyse des résultats}

L'analyse des réseaux sociaux propose « deux ambitions conjointes, qui consistent à la fois à rendre compte des comportements des individus par les réseaux dans lesquels ils s'insèrent, et à rendre compte de la structuration de ces réseaux à partir de l'examen des interactions entre les individus et leurs motivations » (Merklé, 2004). Ce principe ouvre la voie au référent théorique dans lequel s'insère cette étude : la théorie des réseaux sociaux (TRS). Issue notamment des travaux de Ronald Burt (2000) et de Mark Granovetter (2000), cette théorie nous permet de souligner le rôle des réseaux sociaux dans la compréhension du comportement entrepreneurial des femmes depuis le projet de création au développement de leurs activités économiques.

Dès les années soixante-dix, Granovetter avait tenu à souligner que même les comportements individuels qui semblent relever exclusivement de l'«économique » sont influencés par la nature et la structure des réseaux de relations où ces individus se trouvent insérés. Dès lors, de par cette théorie, la constitution du réseau par l'entrepreneur devient une variable d'analyse pertinente pour comprendre l'efficacité de la création d'entreprise (Chabaud \& Ngijol; 2005), sa gestion et son développement.

Le réseau permettant d'évoquer l'idée de connexion, d'échange, d'interaction, de liens et d'alliances (Bassand \& Galland 1993 ; CoenenHuther 1993 ; May 1993), l'approche interactionniste s'est avérée, en outre, pertinente pour l'analyse des données de cette étude. En mettant en avant les interactions entre les acteurs sociaux en réseaux, nous visons par cette approche, à mettre en lumière, les dynamiques individuelles et collectives ainsi que les logiques d'action qui caractérisent nos enquêtées dans l'exercice de leurs activités socio-économiques. Une approche par les réseaux dans une perspective interactionniste, assigne de fait, à cette recherche une mission de compréhension des contraintes émanant de la structure sur les 
comportements des individus qui la composent tout en relevant les relations sociales que ceux-ci entretiennent entre eux.

\section{Résultats}

\subsection{Déterminants de l'adhésion à un réseau}

Les entreprises ou activités informelles se caractérisent généralement par leur vulnérabilité. Leur statut d'activités de subsistance les expose aux aléas de tous genres dont l'une des stratégies résilientes est la constitution des acteurs en réseau. Le rôle important de [distributeurs] ou redistributeurs d'argent, de travail ou de faveurs (Agier, 1983) qui caractérise les réseaux sociaux suscite un engouement chez les « self-employeurs » dont les sources de motivation diffèrent d'un acteur à l'autre. Le tableau ci-dessous présente les raisons d'adhésion des femmes aux réseaux.

Tableau $\mathbf{n}^{\circ} \mathbf{2}$ : Adhésion aux réseaux sociaux : les sources de motivation

\begin{tabular}{|l|c|c|c|c|}
\hline \multicolumn{1}{|c|}{ RAISONS DE L'ADHESION } & $\begin{array}{c}\text { Fréquence } \\
\text { (Rang 1) } \\
\%\end{array}$ & $\begin{array}{c}\text { Fréquence } \\
(\text { Rang 2) } \\
\%\end{array}$ & $\begin{array}{c}\text { Fréquence } \\
(\text { Rang 3) } \\
\%\end{array}$ & $\begin{array}{c}\text { Total } \\
(\%)\end{array}$ \\
\hline Besoin de conseil/soutien & 82,5 & 7,5 & 2,5 & $\mathbf{3 0 , 8}$ \\
\hline Echange d'informations sur les opportunités d'affaires & 12,5 & 57,5 & 10,0 & $\mathbf{2 6 , 7}$ \\
\hline Se faire connaître en tant que professionnel & 0,0 & 7,5 & 10,0 & $\mathbf{5 , 8}$ \\
\hline Être au fait des meilleures pratiques dans ma profession & 0,0 & 5,0 & 2,5 & $\mathbf{2 , 5}$ \\
\hline Recherche de financement & 0,0 & 0,0 & 5,0 & $\mathbf{1 , 7}$ \\
\hline Profiter des activités de formation, de perfectionnement & 2,5 & 0,0 & 0,0 & $\mathbf{0 , 8}$ \\
\hline Encadrement de mes activités & 0,0 & 5,0 & 5,0 & $\mathbf{3 , 3}$ \\
\hline Mieux connaître mes droits/Défendre mes intérêts & 0,0 & 0,0 & 2,5 & $\mathbf{0 , 8}$ \\
\hline Partage d'expériences & 2,5 & 17,5 & 62,5 & $\mathbf{2 7 , 5}$ \\
\hline \multicolumn{1}{|c|}{ TOTAL } & & & $\mathbf{1 0 0}$ \\
\hline
\end{tabular}

Sources : Données d'enquêtes, 2020

Les entrepreneures informelles ont diverses sources de motivation selon les résultats présentés dans le tableau. Par ordre de priorité, trois raisons fondamentales sont à l'origine du choix d'appartenance à un réseau social, il ressort que les besoins de conseil et/ou de soutien occupent le premier rang avec un taux de $82,5 \%$ de citations. Vient ensuite l'échange d'informations sur les opportunités d'affaires avec $57,5 \%$ de citations au rang 2 contre le partage d'expériences au rang 3 totalisant $62,5 \%$. Ces résultats empiriques montrent que la volonté d'adhérer à ces groupes et les interactions sociales qui y ont lieu dénotent de stratégies visant à rendre les activités productives des opératrices informelles plus performantes. Comme l'ont soutenu les adhérentes de l'Union des Femmes Entrepreneures de la Région de Gbêkê lors d'un focus groupe, sans réseau il est difficile d'évoluer dans leur secteur d'activités que 
sont les services aux entreprises. Les marchés passent généralement par les canaux informels (le bouche à oreille). C'est en se frôlant aux autres qu'elles ont non seulement accès à ces informations mais également des stratégies d'acquisition de contrat. La réussite économique de nos enquêtées dépendrait, selon elles-mêmes, de leur insertion dans des réseaux de relations. Cela nous amène à vérifier leur niveau de satisfaction.

Tableau ${ }^{\circ} 3$ : Niveau de satisfaction des enquêtées

\begin{tabular}{|l|c|c|c|}
\hline \multirow{2}{*}{\multicolumn{1}{|c|}{ RAISONS DE L'ADHESION }} & \multicolumn{2}{|c|}{ Satisfaction des membres } & \multirow{2}{*}{ TOTAL (\%) } \\
\cline { 2 - 4 } & Oui $(\mathbf{\%})$ & Non (\%) & \\
\hline Besoin de conseil/soutien & 29,2 & 1,7 & $\mathbf{3 0 , 8}$ \\
\hline Echange d'informations sur les opportunités d'affaires & 25 & 1,7 & $\mathbf{2 6 , 7}$ \\
\hline Me faire connaître en tant que professionnel & 5,8 & 0 & $\mathbf{5 , 8}$ \\
\hline Être au fait des meilleures pratiques dans ma profession & 2,5 & 0 & $\mathbf{2 , 5}$ \\
\hline Recherche de financement & 1,7 & 0 & $\mathbf{1 , 7}$ \\
\hline Profiter des activités de formation, de perfectionnement & 0,8 & 0 & $\mathbf{0 , 8}$ \\
\hline Encadrement de mes activités & 3,3 & 0 & $\mathbf{3 , 3}$ \\
\hline Mieux connaître mes droits/Défendre mes intérêts & 0,8 & 0 & $\mathbf{0 , 8}$ \\
\hline Partage d'expériences & 25,8 & 1,7 & $\mathbf{2 7 , 5}$ \\
\hline \multicolumn{1}{|c|}{ TOTAL } & $\mathbf{9 5}$ & $\mathbf{5}$ & $\mathbf{1 0 0}$ \\
\hline
\end{tabular}

Sources : Données d'enquêtes, 2020

Il ressort de l'enquête de satisfaction de leur appartenance à un réseau en lien avec leurs sources de motivation, que les enquêtées sont très enthousiastes au point où $95 \%$ ont répondu « oui » à la question qui leur a été posée. En tant qu'entrepreneures et/ou créatrices d'activités, ces réseaux sont en phase de répondre aux aspirations des femmes prioritairement relevées dans le tableau, à savoir, le besoin de conseil/soutien (29,2\%), l'échange d'informations sur les opportunités d'affaires (25\%) et le partage d'expériences $(25,8 \%)$. Une enquêtées que nous nommons Fati, commerçante de vivriers témoignait en ces termes :

"Quand je commençais, j'enregistrais des pertes énormes. Depuis que je suis membre de cette association, mon chiffre d'affaires est monté grâce aux conseils que j'ai reçus des anciennes dans le domaine. Elles $m$ 'ont apprise à conserver mes produits et à les reconditionner».

L'opinion favorable de nos enquêtées au sujet de leurs sources de motivation vient étayer l'approche défendue par Chabaud et Ngijol (2005) selon laquelle le réseau est lui-même constitué par l'entrepreneur afin d'appuyer son effort de création d'entreprise. Sans en avoir les compétences, 
à priori, les groupes d'appartenance joueraient un rôle d'accompagnement de leurs membres dans la mobilisation de ressources de tous genres. Au regard du potentiel de transferts de savoir et d'avantages divers qu'elles représentent, quelques actions concrètes de ces réseaux au profit de leurs adhérentes ont été mises en évidence.

\subsection{Accompagnement des activités et/ou entreprises par les réseaux}

Satisfaites de leur adhésion à leur réseau d'appartenance en lien avec les raisons qui les y ont poussées, un facteur non moins négligeable caractérisant les entrepreneures informelles et leurs activités est l'accompagnement en temps réel de leurs activités.

Tableau $n^{\circ} \mathbf{4}$ : Nature des accompagnements et leur impact sur les activités

\begin{tabular}{|l|c|c|c|c|c|}
\hline \multirow{2}{*}{\multicolumn{1}{|c|}{ Accompagnements de l'activité }} & \multicolumn{3}{c|}{ Impact (\%) } & TOTAL \\
\cline { 2 - 6 } & $\begin{array}{c}\text { Aucun } \\
\text { impact }\end{array}$ & Positif & Négatif & Mitigé & $(\mathbf{\%})$ \\
\hline Aides financières & 5 & 20 & 5 & 0 & $\mathbf{3 0}$ \\
\hline Formations & 12,5 & 2,5 & 0 & 0 & $\mathbf{1 5}$ \\
\hline Coaching personnalisé & 17,5 & 15 & 2,5 & 2,5 & $\mathbf{3 7 , 5}$ \\
\hline $\begin{array}{l}\text { Accompagnement à la création/reprise } \\
\text { d'entreprise }\end{array}$ & 2,5 & 5 & 0 & 0 & $\mathbf{7 , 5}$ \\
\hline Crédit-bail & 2,5 & 2,5 & 0 & 0 & $\mathbf{5}$ \\
\hline Apport en clientèle & 0 & 2,5 & 0 & 0 & $\mathbf{2 , 5}$ \\
\hline Assistance sociale & 0 & 2,5 & 0 & 0 & $\mathbf{2 , 5}$ \\
\hline \multicolumn{1}{|c|}{ TOTAL } & $\mathbf{4 0}$ & $\mathbf{5 0}$ & $\mathbf{7 , 5}$ & $\mathbf{2 , 5}$ & $\mathbf{1 0 0}$ \\
\hline
\end{tabular}

Sources : Données d'enquêtes, 2020

En situations difficiles, le groupe est amené à apporter assistance aux membres. Les données empiriques du tableau $\mathrm{n}^{\circ} 4$ relèvent, à cet effet, un certain nombre d'actions collectives ou individuelles dont ont bénéficié des adhérentes. En outre, les données montrent l'impact de ces accompagnements sur l'activité des bénéficiaires en vue d'en apprécier l'efficacité pour cellesci. Dans l'ensemble, $37,5 \%$ des enquêtées ont bénéficié de coaching personnalisé contre $30 \%$ d'aides financières. L'activité informelle étant caractérisée par le manque de professionnalisme des acteurs généralement moins instruits, ils apprennent sur le tas et se lancent quasi exclusivement dans une activité sans en connaitre les contours, sans une formation professionnelle à même de leur transmettre des savoirs techniques. Du coup, se faire accompagner par des pairs plus expérimentés est une belle opportunité de suivi, de conseils. En plus du manque de savoir-faire, un problème épineux pour les entrepreneures informelles est le manque de ressources financières. Le capital financier est indispensable tant en phase de création que de post- 
création. Pour des micros et très petites entreprises qui ne sont pas solvables auprès des banques, se faire accompagner par un réseau est une stratégie résiliente.

Sur ce sujet, la présidente de l'Union des femmes couturières de Bouaké s'exprimait en ces termes :

"les femmes créatrices de Très Petites entreprise (TPE) sont confrontées à l'absence d'aide financière, de soutien des institutions financières. Par nos actions de solidarité, elles retrouvent confiance en leur potentiel d'entrepreneures. Notre groupement les accompagne ainsi à travers les fonds mobilisés par les cotisations mensuelles, les tontines et nos sponsors à l'installation de leur atelier et/ou à l'achat de machines à coudre ou tout autre équipement pour certaines et la formation pour d'autres ».

Les appréciations de l'impact de ces modes d'accompagnement sur les activités diffèrent néanmoins selon les données du tableau. Quel que soit le mode d'accompagnement, dans l'ensemble, les avis des enquêtées varient entre celles qui ne perçoivent « aucun impact » $(40 \%)$ et celles pour qui il y a « un impact positif» $(50 \%)$ sur leurs activités. Bien que la différence ne soit pas considérable, on note une amélioration dans la pratique des activités. Cela nous amène à questionner les revenus mensuels tirés par les enquêtées depuis leur adhésion à leur réseau.

Tableau $\mathbf{n}^{\circ} \mathbf{5}$ : Revenus mensuels des activités après adhésion au réseau

\begin{tabular}{|c|c|c|c|c|}
\hline \multirow[b]{2}{*}{$\begin{array}{l}\text { Revenu mensuel en FCFA } \\
\text { avant l'adhésion }\end{array}$} & \multicolumn{3}{|c|}{ Revenu mensuel en FCFA après adhésion (\%) } & \multirow[b]{2}{*}{ TOTAL } \\
\hline & $\begin{array}{c}\text { Aucune } \\
\text { amélioration }\end{array}$ & {$[150000,250000[$} & {$\left[\begin{array}{c}250000,350000 \\
{[}\end{array}\right.$} & \\
\hline Aucun revenu & 17,5 & 12,5 & 0 & 30 \\
\hline Moins de 30000 & 20 & 25 & 0 & 45 \\
\hline$[30000,60000[$ & 2,5 & 15 & 0 & 17,5 \\
\hline$[60000,120000[$ & 2,5 & 2,5 & 2,5 & 7,5 \\
\hline TOTAL & 42,5 & 55 & 2,5 & 100 \\
\hline
\end{tabular}

Sources : Données d'enquêtes, 2020

Même s'il n'est pas le seul, le revenu est un indicateur important dans l'appréciation des conditions de vie et de travail. En mettant en rapport les revenus avant et ceux après l'adhésion, il ressort que 42,5\% des enquêtées, quel que soit leur revenu avant d'adhérer au réseau n'ont observé aucune amélioration au niveau de leur revenu après avoir y adhéré, contre 55\% dont le revenu s'est amélioré à hauteur de 150000 à 250000 fcfa. Ces chiffres nous inspirent qu'il y ait une possibilité d'amélioration soutenue des conditions de vie et de travail grâce à la synergie des efforts favorisée par le réseau. Plusieurs 
cas de figures se présentent dans ce tableau. Le facteur confiance prévaut dans un réseau, celles qui n'ont aucun moyen financier donc aucun revenu ne s'appuie sur la générosité du groupe pour lancer une affaire en prenant des marchandises à crédit par exemple. Certaines par contre, passent par le biais des tontines qu'elles prennent en premier quand d'autres s'endettent auprès de la trésorerie du réseau pour renforcer leur capital. Si la situation financière semble s'améliorer ou est restée identique avec l'adhésion à un réseau, qu'en est-il des retombés réels sur les activités ?

Tableau $\mathbf{n}^{\circ} \mathbf{6}$ : Les retombés des accompagnements sur les activités

\begin{tabular}{|c|c|c|c|c|c|c|c|}
\hline \multirow[b]{2}{*}{ Secteur d'activité } & \multicolumn{6}{|c|}{ Retombés de l'accompagnement sur l'activité (\%) } & \multirow[b]{2}{*}{ TOTAL } \\
\hline & $\begin{array}{l}\text { Améliora } \\
\text { tion des } \\
\text { marges }\end{array}$ & $\begin{array}{c}\text { Améliora } \\
\text { tion des } \\
\text { ventes }\end{array}$ & $\begin{array}{l}\text { Augmentat } \\
\text { ion du } \\
\text { capital }\end{array}$ & $\begin{array}{c}\text { Ouverture aux } \\
\text { nouveaux } \\
\text { marchés }\end{array}$ & $\begin{array}{l}\text { Amélioration de } \\
\text { la qualité des } \\
\text { produits/services }\end{array}$ & Aucun & \\
\hline $\begin{array}{l}\text { Commerce de } \\
\text { gros et/ou détails }\end{array}$ & 9 & 9 & 5,6 & 2,2 & 1,1 & 3,4 & $\mathbf{3 0 , 3}$ \\
\hline Agroalimentaire & 4,5 & 4,5 & 4,5 & 0 & 0 & 4,5 & 18 \\
\hline Restauration & 2,2 & 2,2 & 2,2 & 0 & 0 & 0 & 6,7 \\
\hline Industries & 0 & 0 & 0 & 0 & 0 & 1,1 & 1,1 \\
\hline $\begin{array}{l}\text { Cosmétique/soin/ } \\
\text { beauté }\end{array}$ & 5,6 & 5,6 & 4,5 & 0 & 1,1 & 1,1 & 18 \\
\hline Couture, mode & 5,6 & 5,6 & 6,7 & 2,2 & 0 & 5,6 & 25,8 \\
\hline TOTAL & 27 & 27 & 23,6 & 4,5 & 2,2 & 15,7 & 100 \\
\hline
\end{tabular}

Sources : Données d'enquêtes, 2020

L'analyse des retombés des accompagnements par les réseaux trouve sa pertinence sous la loupe des activités. Bien qu'ils soient limités professionnellement et à la limite de l'informel, les différents réseaux qui ont fait l'objet de cette étude, contribuent naturellement à l'épanouissement des activités de leurs membres. Les apports de ces réseaux aux femmes créatrices et entrepreneures transparaissent dans ce tableau avec $27 \%$ d'amélioration des marges, $27 \%$ d'amélioration des ventes et $23,6 \%$ d'augmentation du capital social tous les secteurs d'activités confondus. Les réseaux et les relations sociales qui s'y développent s'avèrent, de fait, décisifs dans la croissance et la pérennisation des très petites entreprises. Ces données corroborent l'analyse faite plus haut sur les formes d'accompagnement. Nous savons que les activités concernées sont généralement précaires, vulnérables car entourées d'incertitudes tant dans leur création que dans leur gestion; les relations interpersonnelles qui se tissent au sein des réseaux et dans lesquelles s'encastre l'entrepreneure constitue un terrain riche d'informations, d'opportunités et de ressources à disposition de celle-ci dont l'accès serait quasi impossible si elle était seule isolée. Ce capital social que constitue le 
réseau, en procurant différents moyens utiles à l'entrepreneure devient par ailleurs un instrument de sécurité grâce à la réciprocité et la coopération au sein des relations.

\subsection{Sécurité des activités : le rôle des réseaux sociaux}

Sans être de véritables professionnels de l'accompagnement, les réseaux à l'étude se présentent comme une assurance pour la pérennité des entreprises de leurs adhérentes. En en améliorant les performances, la croissance et la stabilité, ils assurent la sécurité de ces entreprises. Les niveaux de sécurité procurés par ces réseaux sont présentés dans le tableau ci-après.

Tableau n ${ }^{\circ} 6$ : Niveaux de sécurité des micros et petites entreprises selon les réseaux

\begin{tabular}{|l|c|c|c|c|}
\hline \multirow{2}{*}{\multicolumn{1}{|c|}{ Réseau d'appartenance }} & \multicolumn{2}{c|}{ Niveau de sécurité de votre activité (\%) } & \multirow{2}{*}{ TOTAL } \\
\cline { 2 - 4 } & Faible & Moyen & Elevé & \\
\hline $\begin{array}{l}\text { Union des Femmes Entrepreneures de la Région de } \\
\text { Gbèkè (UFERG) }\end{array}$ & 2,5 & 7,5 & 15 & $\mathbf{2 5}$ \\
\hline Union des femmes couturières de Bouaké (UFCB) & 0 & 7,5 & 17,5 & $\mathbf{2 5}$ \\
\hline $\begin{array}{l}\text { Coopérative des Femmes du Secteur du Vivrier de } \\
\text { la Région du Gbêkê }\end{array}$ & 2,5 & 2,5 & 17,5 & $\mathbf{2 2 , 5}$ \\
\hline Association Sœurs Unies d'Ahougnassou & 0 & 7,5 & 20 & $\mathbf{2 7 , 5}$ \\
\hline \multicolumn{1}{|c|}{ TOTAL } & $\mathbf{5}$ & $\mathbf{2 5}$ & $\mathbf{7 0}$ & $\mathbf{1 0 0}$ \\
\hline
\end{tabular}

Sources : Données d'enquêtes, 2020

Le niveau de sécurité est généralement positif comme le présente le tableau. 70\% des enquêtées membres des réseaux consultés reconnaissent que le niveau de sécurité de leurs activités est élevé depuis leur adhésion à leur structure d'appartenance contre $25 \%$ dont le niveau de sécurité est moyen. Globalement, les sources de motivation des adhérentes sont justifiées comme nous l'avons mentionné à l'entame de ce rapport d'enquête. Le témoignage suivi est révélateur à cet effet.

"Quand j'ai ouvert mon atelier de couture, je ne croyais pas en moi. Je ne m'en sortais pas et au bout du premier trimestre j'étais à deux doigts de fermer à cause des charges de fonctionnement qu'il m'était quasiment impossible de couvrir. Heureusement pour moi j'ai intégré l'Union des couturières qui m'a aidé à sortir peu à peu la tête de l'eau. J'ai pris confiance en moi d'abord par mes créations en apprenant par la suite à les vendre. Depuis maintenant 3 ans, mon atelier est plus que stable » (Mme Koffi).

Cela n'est pas fortuit au regard de la dénomination de ces réseaux. Ils sont, à l'exception de la dernière ligne du tableau, des groupements «professionnels » spécialisés dans un secteur d'activités précis. Sachant que 
les entrepreneur(e)s sont exposé(e)s ou confronté(e)s constamment à des problèmes de management, de financement complexes...qui conditionnent leurs activités dans la précarité, ces réseaux permettent aux membres de se rassembler pour se sécuriser. A la différence des réseaux professionnels d'accompagnement, ces groupes pour le moins informels, constituent des cadres par excellence d'échanges entre pairs sur les questions de diverses natures telles l'exploitation d'opportunités d'affaires, la mobilisation de ressources financières et/ou matérielles, la connaissance de son secteur d'activité et/ou de son marché. Les attentes et résultats de ces échanges procurent aux acteurs un sentiment de sécurité. Ainsi, les facteurs au sein des réseaux qui procurent la sécurité aux membres de par leurs activités ont été analysés. Voyons la situation sécuritaire sous la loupe de l'ancienneté dans les activités.

Tableau n $\mathbf{n}^{\circ}$ : Le niveau de sécurité de l'activité sous l'angle de l'ancienneté

\begin{tabular}{|l|c|c|c|c|}
\hline \multirow{2}{*}{ Ancienneté dans l'activité } & \multicolumn{2}{|c|}{ Niveau de sécurité de votre activité ou entreprise(\%) } & \multirow{2}{*}{ TOTAL } \\
\cline { 2 - 5 } & Faible & Moyen & Elevé & \\
\hline Moins d'1 an & 5 & 0 & 0 & $\mathbf{5}$ \\
\hline 1 à 5 ans & 0 & 7,5 & 5 & $\mathbf{1 2 , 5}$ \\
\hline 5 à 10 ans & 2,5 & 2,5 & 17,5 & $\mathbf{2 2 , 5}$ \\
\hline 10 à 15 ans & 0 & 5 & 20 & $\mathbf{2 5}$ \\
\hline Plus de 15 ans & 5 & 10 & 20 & $\mathbf{3 5}$ \\
\hline \multicolumn{1}{|c|}{ TOTAL } & $\mathbf{1 2 , 5}$ & $\mathbf{2 5}$ & $\mathbf{6 2 , 5}$ & $\mathbf{1 0 0}$ \\
\hline
\end{tabular}

Sources : Données d'enquêtes, 2020

La sécurité d'une entreprise/activité se justifie par sa pérennité. Cela implique que celle-ci ait survécu aux risques de sa disparition. Dans le cadre de cette étude, l'indicateur de cette pérennité est l'ancienneté dans l'exercice de l'activité. Une comparaison entre les entreprises pérennes et celles disparues aurait enrichit l'analyse et la pertinence de la corrélation établie entre les variables présentés dans le tableau ci-dessus. En ce qui concerne les données, la longévité des activités est justifiée par le facteur sécurité. Les statistiques le montrent si bien que le niveau de sécurité est élevé au fur et à mesure que l'activité gagne en maturité. Autant les activités de plus de 15 ans sont les plus nombreuses avec un taux de 35\% autant elles cumulent $30 \%$ de niveau de sécurité moyen (10\%) et élevé (20\%). Bien qu'elles restent vulnérables avec un risque plus important de disparaitre comparativement à une grande entreprise, leur performance est justifiée avec le concours des réseaux. Le groupe a donc un impact positif sur l'entreprise à travers l'accompagnement. Il n'est pas le seul facteur bien entendu d'autant plus que la réussite en entrepreneuriat relève d'un contexte plus global impliquant par 
ailleurs des critères de secteur d'activité, du profil de son créateur, de l'investissement consenti au démarrage, etc., mais il est déterminant aux différentes phases de progression de l'entreprise. En effet, la création d'une entreprise nécessite des compétences multiples qu'un(e) créateur/trice ne peut maîtriser qu'avec le concours de ses pairs plus expérimentés ou vivant les mêmes réalités que lui/elle. De par les relations interpersonnelles, les acteurs socioéconomiques informels articulent ainsi les dimensions économiques et sociales reposant sur une logique de réciprocité et de redistribution. Au-delà de cette vision idéalisée ou angélique des relations interpersonnelles, subsistent néanmoins des tensions pouvant caractériser ces acteurs sociaux entrant en relation dans les réseaux sociaux. Cette dimension des rapports sociaux a été fondamentale dans nos analyses.

\subsection{Tensions inhérentes aux relations au sein des réseaux}

S'ils sont relativement efficaces dans l'assistance des activités de leurs membres, les réseaux sociaux ne sont toutefois pas à l'abri des tensions. Ces conflits se présentent sous diverses formes. Le tableau ci-après en donne quelques-unes.

Tableau $\mathbf{n}^{\circ} \mathbf{8}$ : Tensions au sein de l'association

\begin{tabular}{|l|c|c|c|}
\hline \multirow{2}{*}{ Natures des tensions } & \multicolumn{2}{c|}{$\begin{array}{c}\text { Tensions au sein de } \\
\text { l'association (\%) }\end{array}$} & \multirow{2}{*}{ TOTAL } \\
\cline { 2 - 4 } & Oui & Non & \\
\hline Non réponse & 0 & 10 & $\mathbf{1 0}$ \\
\hline Problèmes de leadership & 27,5 & 0 & $\mathbf{2 7 , 5}$ \\
\hline Problèmes de gestions de fonds & 22,5 & 5 & $\mathbf{2 7 , 5}$ \\
\hline $\begin{array}{l}\text { Différences d'opinions ou de points de vue } \\
\text { violentes }\end{array}$ & 5 & 0 & $\mathbf{5}$ \\
\hline Favoritisme et traitement inégalitaire & 15 & 2,5 & $\mathbf{1 7 , 5}$ \\
\hline Critiques négatives & 7,5 & 5 & $\mathbf{1 2 , 5}$ \\
\hline \multicolumn{1}{|c|}{ TOTAL } & $\mathbf{7 7 , 5}$ & $\mathbf{2 2 , 5}$ & $\mathbf{1 0 0}$ \\
\hline
\end{tabular}

Sources : Données d'enquêtes, 2020

La vie d'une association n'est pas un fleuve tranquille. Les réseaux de solidarité relativement appréhendée comme stratégie résiliente des microentrepreneures face aux risques liés à leurs activités sont généralement confrontés à des situations conflictuelles en leur sein. Les données empiriques contenues dans ce tableau le confirment avec un taux de 77,5\%. La nomenclature des conflits présentés est largement dominée par les problèmes de leadership $(27,5 \%)$ et de gestion de fonds $(22,5 \%)$. Les problèmes de leadership tournent couramment autour des difficultés persistantes en matière de gouvernance, de même que les problèmes liés à la personnalité du créateur 
du réseau ou de ses responsables. La question de gouvernance transparaît par ailleurs dans une autre source de conflits mentionnée par les enquêtées à savoir le favoritisme et le traitement inégalitaire des membres de l'association à un taux de $15 \%$.

La gestion de fonds est un élément décisif de la cohésion du groupe. Quand les fonds sont mal gérés ils sont sources de division. Dans un contexte où les membres sont divisés sur l'autel de la mauvaise gestion des ressources, il est difficile d'envisager une amélioration des relations. En conséquence, les adhérents qui s'intègrent dans le réseau, sont moins engagés. Ces tensions ont probablement des liens avec les problèmes mentionnés ci-après.

Tableau $\mathbf{n}^{\circ} \mathbf{9}$ : Les difficultés du réseau

\begin{tabular}{|c|c|}
\hline Les difficultés du réseau & Fréquences $(\%)$ \\
\hline Manque de crédibilité & 6,3 \\
\hline Désistement des membres & 6,3 \\
\hline Problèmes de trésorerie & 37,5 \\
\hline Manque de communication & 22,5 \\
\hline Structure tourne en rond & 18,8 \\
\hline Inefficacité dans la gestion des projets des adhérentes & 8,8 \\
\hline TOTAL & 100 \\
\hline
\end{tabular}

Sources : Données d'enquêtes, 2020

Les problèmes de nature à remettre en cause la pérennité d'un réseau sont de divers ordres. Les plus courants, citées par les enquêtées, sont prioritairement les problèmes de trésorerie (37,5\%), le manque de communication $(22,5 \%)$ et le fait que la structure tourne en rond $(18,8 \%)$ sans objectifs ou objets concrets. Les difficultés financières récurrentes dans les associations sont dues généralement au fait qu'elles dépendent de dons et de cotisations de leurs membres d'autant qu'elles sont des organisations à but non lucratif. La situation devient plus difficile voire compliquée lorsque les ressources sont mal gérées ou destinées aux activités qui ne servent pas les intérêts du groupe. Cela a été souligné par les enquêtées un peu plus haut. Un autre problème handicapant pour les réseaux est la communication lorsqu'elle est quasi inexistante ou de mauvaise qualité. Les retentions d'informations, les retards dans la diffusion d'informations, les erreurs dans le choix des cibles...sont de nature à détruire la cohésion et la solidarité au sein de l'association. Tout cela aboutit à une situation qui fait tourne l'association en rond. 


\section{Discussion}

\subsection{Déterminants de l'adhésion à un réseau}

Ce point de notre analyse a révélé trois principales sources de motivation chez les entrepreneures informelles dans leur volonté d'appartenir à un réseau, à savoir le besoin de conseil/soutien, l'échange d'informations sur les opportunités d'affaires et le partage d'expériences. Une enquête réalisée par Verdoni (2014) nous instruit sur les mêmes résultats. En s'appuyant sur trois facteurs motivant, l'auteur soutient, en effet, que les chefs d'entreprise se décident à rejoindre un réseau pour un souci d'appui stratégique et méthodologique fourni par la tête de réseau, pour accéder à la visibilité et la connaissance du marché, et pour accéder aux échanges intra réseau. Webb et al. (2009) le soulignent également quand ils disent que l'insertion au sein de communautés est favorable au développement des activités entrepreneuriales informelles car ces communautés se substituent aux institutions formelles. De part ces sources de motivation, les réseaux sociaux et communautés constituent dès lors des déterminants essentiels de la dynamique courante des activités informelles et de la réussite des entrepreneurs (Berrou, 2014). Les activités qui s'exercent au sein de ces réseaux sont analysées, au final, dans une perspective de l'économie sociale et solidaire (Castel, 2007). Ces réseaux de relations interpersonnelles permettent alors l'accès à diverses ressources (information, conseils, soutien financier, recrutement, etc.) essentielles dans la mise en œuvre courante des activités entrepreneuriales (Berrou, Idem).

\subsection{Accompagnement des activités et/ou entreprises par les réseaux}

L'analyse des données issues de notre enquête a révélé que 37,5\% des enquêtées ont bénéficié de coaching personnalisé contre $30 \%$ d'aides financières. Les réseaux étudiés sont d'un grand apport pour le développement des activités de leurs membres. Ces soutiens des réseaux sont également soulignés par Pluchart (2013) qui constate que les ressources attendues d'un réseau d'accompagnement doivent permettre au créateur d'affaires des accès aux partenaires, aux financements, aux fournitures et aux débouchés, à la formation, au marché de l'emploi, aux brevets et marques, aux locaux, équipements et matériels... nécessaires afin de contribuer à la construction des avantages concurrentiels de son entreprise. Ces éléments constitutifs de l'accompagnement viennent répondre aux interrogations de Cuzin et alii (2004) relatives aux dimensions structurantes de l'accompagnement entrepreneurial. Toutefois, l'efficacité de cet accompagnement sur les entreprises affiliées à ces réseaux ne semble pas évidente pour certains chercheurs. Leger-Jarniou et Saporta (2006) remettent ainsi en question la pertinence de l'accompagnement en situation entrepreneuriale des réseaux sociaux. D'autres facteurs externes peuvent influer sur le processus d'accompagnement et affecter son efficience, comme la conjoncture 
économique ou la zone d'accueil de la nouvelle entreprise. Dans le cadre de notre étude nous avons évalué l'impact de ces réseaux sur la performance des activités de nos enquêtées afin d'en savoir davantage. Il ressort de nos investigations que l'impact des apports de ces réseaux aux femmes créatrices et entrepreneures est positif dans l'ensemble. Nous avons pu constater des améliorations au niveau des marges, des ventes et une augmentation du capital, tous les secteurs d'activités confondus. Au regard de ces notes positives, un aperçu de la sécurité des activités s'est avéré nécessaire.

\subsection{Sécurité des activités : le rôle des réseaux sociaux}

L'amélioration de la performance, la stabilité et la croissance d'une activité/entreprise en déduisent sa sécurité. Pour ce qui concerne notre étude, les données empiriques collectées stipulent un niveau de sécurité élevé des activités dans l'ensemble. Mais notre position n'est pas partagée par Tognini (2017) pour qui, il faut savoir resauter. Pour lui il ne faut pas être partout tout le temps ; il faut savoir sélectionner les réseaux auxquels participer ; analyser le coût/efficacité de la participation aux réseaux ; penser au facteur temps. Un dirigeant qui passe plus de $10 \%$ de son temps à resauter se mettrait en danger. Il faut savoir être au bon endroit au bon moment et, surtout, ne pas tomber dans l'excès de ne plus être présent dans son entreprise.

Inscrivant son étude dans un contexte plus global de jeux d'alliances entre des organisations, des PME, Parkhe (1993) précisait ainsi qu'un des enjeux majeurs est de limiter les risques relationnels inhérents à ces opérations caractérisées par une incertitude quant au comportement futur des partenaires. Pour leur part, Das et Teng (1996) soutiennent qu'une alliance peut être confrontée à deux types de risques : celui lié à la performance et le risque relationnel. Hatfield et Pearce (1994) présentent les tensions à l'intérieur des réseaux d'une autre manière. Pour eux, des attentes non réalistes vis-à-vis de l'alliance ou un manque de congruence des objectifs de chacun des partenaires peut conduire à une instabilité forte. Les alliés peuvent par exemple avoir des visions différentes de la durée de l'alliance (souvent ambiguë), certains faisant preuve d'impatience dans la réalisation des objectifs.

\subsection{Tensions inhérentes aux relations au sein des réseaux}

Nos analyses nous ont permis de mettre en exergue, la présence des tensions au sein des réseaux avec un taux de 77,5\%. Dans la nomenclature des tensions présentées, la part belle est faite aux problèmes de leadership $(27,5 \%)$ et de gestion de fonds (22,5\%). Après avoir remarqué que les différentes tensions qui animent la dynamique d'un réseau sont l'expression de la fragilité des liens tissés entre les différents intervenants, Libois et Loser (2010) ont pu observer les tensions relevant de la hiérarchie des métiers, mais également des rapports de pouvoir entre professionnels occupant des fonctions 
institutionnelles différentes. Pour eux, ces tensions constituent une gageure de taille pour les pratiques en réseau. Michaud (2011) ne dira pas le contraire quand elle a soutenu dans son étude sur les coopératives, que les tensions étaient inhérentes aux organisations. Des difficultés relationnelles peuvent se manifester (désaccords quant aux objectifs de l'alliance, manque d'engagement des partenaires, etc.) et déboucher sur des conflits et une rupture prématurée de l'accord (Puthod et Thévenard-Puthod, 2006).

\section{Conclusion}

A travers cette étude nous avons essayé de cerner les rapports pouvant exister entre les réseaux sociaux et le développement des micros et petites entreprises ou activités relevant du secteur informel. Pour ce faire, nous avons pris pour prétexte, les femmes exerçant dans ce secteur. L'objectif général poursuivi était de comprendre les logiques qui sous-tendent les formes de réseaux sociaux mobilisés par les femmes dans l'exercice de leurs activités afin de déterminer dans quelle mesure ceux-ci leur permettent de développer leurs activités. La collecte de données empiriques de cette étude a lieu à Bouaké au centre de la Côte d'Ivoire auprès d'un échantillon de 96 enquêtées appartenant à quatre associations. La constitution de l'échantillon s'est faite à l'aide de la technique d'échantillonnage par grappes d'abord pour le choix du groupe d'appartenance puis l'échantillonnage aléatoire simple pour le choix des participantes. Les données obtenues par une approche mixte (qualitative et quantitative) ont été analysées au moyen de la méthode interactionniste. L'analyse a révélé trois principaux déterminants dans la volonté des entrepreneures informelles d'appartenir à un réseau, à savoir le besoin de conseil/soutien, l'échange d'informations sur les opportunités d'affaires et le partage d'expériences. Dans ces réseaux, elles ont bénéficié d'accompagnement au profit du développement de leurs activités en termes de coaching personnalisé, d'aides financières, de formation. Il en ressort que cet accompagnement a contribué à l'amélioration de la performance, la stabilité et la croissance des activités concernées garantissant in fine leur sécurité. Ces résultats positifs n'occultent pas pour le moins qu'on puisse souligner la présence de tensions dans ces réseaux. Lesquelles tensions ont été notifiées par les enquêtées elles-mêmes confrontées dans leurs relations avec les autres membres de leur réseau respectif aux problèmes de leadership, de critiques acerbes et de gestion de fonds.

\section{References:}

1. Agier, M. (1983). Commerce et sociabilité: les négociants soudanais du quartier Zongo de Lomé, Paris, ORSTOM, 318p. 
2. Anderson, S. \& Baland, J.-M. (2002). "The Economics of Roscas and Intrahousehold Resource Allocation", Quarterly Journal of Economics, 117 (3): 963-95.

3. Baland, J.-M., Guirkinger C. \& Mali C. (2007). "Pretending to be Poor: Borrowing to Escape Forced Solidarity in Cameroon", Mimeo.

4. Bassand, M. \& Galland B. (1993). "Dynamique des réseaux et société ». FLUX Cahiers scientifiques internationaux Réseaux et Territoires, 13-14, 7-10.

5. BERROU, J-P. (2014). " Les entrepreneurs du secteur informel" In Dictionnaire sociologique de l'entrepreneuriat, Sciences Po. Les Presses, pp.227-243.

6. BIT (2013). La transition de l'économie informelle vers l'économie formelle, rapport V (1), Conférence internationale du Travail, 103e session, Genève, 2014 (Genève).

7. BIT (2016). Les femmes au travail: tendances 2016, Genève.

8. BIT (2000). Emploi et protection sociale dans le secteur informel, 277è Session, Génève, 19 p.

9. Burt, R.S. (2000). « The network structure of social capital », dans R.I. Sutton et B. Staw (dir.), Research in Organizational Behavior, 22, Greenwich, Conn., JAI Press.

10. Castel, O. « De l'économie informelle à l'économie populaire solidaire : Concepts et pratiques », dans Crevoisier, O., Hainard F. \& Ischer P. (dir.) (2007). L'économie informelle : une alternative à l'exclusion économique et sociale?, Berne, Unesco et Université de Neuchâtel, pp. 111-134.

11. Chabaud, D. \& Ngijol, J. (2005). «La contribution de la théorie des réseaux sociaux à la reconnaissance des opportunités de marché » in Revue internationale P.M.E., 18 (1), 29-46.

12. Coenen-Huther, J. (1993). «Réseaux et sociologie». FLUX Cahiers scientifiques internationaux Réseaux et Territoires, 13-14, 32-40.

13. Cuzin, R. \& Fayolle A. (2004). « Les dimensions structurantes de l'accompagnement en création d'entreprise », La Revue des Sciences de Gestion, $\mathrm{N}^{\circ} 210$, pp. 77-88.

14. Das, T. K. et Teng, B. S. (1996). "Risk types and inter-firm alliance structures", Journal of Management Studies, vol. 33, n 6, p. 827-843.

15. Di falco, S. \& Bulte E. (2010). "Social Capital and Weather Shocks in Ethiopia: Climate Change and Culturally-induced Poverty Traps", Mimeo, LSE et Wageningen University.

16. Fofana, V., Adou, P. V., \& Camara, M. (2020). «Dynamique Entrepreneuriale Des Femmes En Côte D'ivoire : Une Etude De Cas Des Femmes Chefs De Ménage » In World Wide Journal of Multidisciplinary Research and Development, pp 63-76. 
17. Fofana, V. (2017). « Microfinance Et Mobilisation De L'épargne Dans Le Secteur Informel. Le Cas Des Commerçantes De Vivriers De Bouake » In European Scientific Journal, Vol.13, PP 50-71.

18. Gabriella, R. (2015). Une étude sur les femmes dans l'économie informelle dans trois districts d'Ethiopie, https://www.ituccsi.org/une-etude-sur-les-femmes-dans-l?lang=en, consulté le 28/08/2020.

19. Gning, S. B. (2013). « Les femmes dans le secteur informel aujourd'hui », in Travail et genre dans le monde. L'état des savoirs, (sous dir) Margaret Maruani, La Découverte, pp. 336-344.

20. Granovetter, M. (2000). Le marché autrement. Les réseaux dans l'économie, Paris, Desclée de Brouwer.

21. Hoff, K. \& Sen, A. (2006). "The Kin as a Poverty Trap", in Bowles, S., Durlauf, S.N. \& K. Hoff (Eds), Poverty Traps, Princeton University Press, New York.

22. Hunt, A. \& Samman, E. (2016). Women's economic empowerment: Navigating enablers and constraints, ODI Development Progress research report Londres, Overseas Development Institute (ODI).

23. Kabeer, N. \& Natali, L. (2013). "Gender equality and economic growth: Is there a win-win?" Working Paper No. 417 (Brighton, Institute of Development Studies).

24. L'écuyer, R. (1990). Méthodologie de l'analyse développementale des contenus. Méthode GPS et concept de soi. Québec : Presses de l'Université du Québec, $490 \mathrm{p}$.

25. Leger-Jarniou, C. \& Saporta, B. (2006). « Editorial: L'accompagnement en situation entrepreneuriale: pertinence ou cohérence », Revue de l'Entrepreneuriat, Vol 5, №1, pp. 9-12.

26. Libois, J. \& Lose, F. (2010). Travailler en réseau: Analyse de l'activité en partenariat dans les domaines du social, de la santé et de la petite enfance. Nouvelle édition [en ligne]. Genève : Éditions ies, 230p.

27. Marie, A., Vuarin, R., Leimdorfer, F. \& al. (2008). L'Afrique des individus. Itinéraires citadins dans l'Afrique contemporaine (Abidjan, Bamako, Dakar, Niamey). Éditions Karthala, «Hommes et sociétés », 442p.

28. May, N. (1993). "Organisation productive et réseaux ». FLUX Cahiers scientifiques internationaux Réseaux et Territoires 13-14 pp. 19-32.

29. Mbueselir, S. A. (2010). Economie informelle et réseaux sociaux à Kinshasa, Université Catholique de Louvain, 391p.

30. Merckle, P. (2004). La sociologie des réseaux sociaux. Paris, Coll. "Repères", La Découverte. 
31. Michaud V. (2011). «Proposition pour l'étude des tensions dans le mouvement, la sociomatérialité et le paradoxe », Communiquer, 5, 4774.

32. ONU-Femmes (Entité des Nations Unies pour l'égalité des sexes et l'autonomisation de la femme) (2015). Progress of the World's Women 2015-16: Transforming economies, realizing rights (New York, NY).

33. OIT (2018). Autonomisation des femmes qui travaillent dans l'économie informelle, Génève, $19 \mathrm{p}$.

34. OIT (2019). Femmes et hommes dans l'économie informelle: Un panorama statistique, (troisième édition) / Genève, Bureau international du Travail, $170 \mathrm{p}$.

35. Parkhe, A. (1993). "Strategic alliances structuring: a game theory and transaction cost examination of interfirm cooperation", Academy of Management Journal, vol. 36, $\mathrm{n}^{\circ}$ 4, p. 794-829.

36. Platteau, J.-P. (2000). Institutions, Social Norms and Economic Development, Harwood Academic Publishers, Amsterdam.

37. Pluchart, J.-J. (2013). «Les performances des réseaux d'accompagnement entrepreneurial » In ANDESE | «Vie \& sciences de l'entreprise $\gg \mathrm{N}^{\circ} 193$, pp 93-113.

38. Puthod, D. \& Thevenard-Puthod, C. (2006). «Coopération, tensions et conflit dans un réseau d'innovation construit autour d'une PME », Revue française de gestion, vol. ${ }^{\circ}$ 164, no. 5, pp. 181-204.

39. Simen, S. F. (2018). Dynamique de l'entrepreneuriat informel au Sénégal : caractéristiques et particularités, IMS, Jun 2018, Montpellier, France. Halshs-01782037.

40. Tognini, F. (2017). «Celui qui fréquente les réseaux professionnels détient l'information avant qu'elle ne soit diffusée sur les réseaux sociaux», I2D - Information, données \& documents, (Volume 54), p. 67-67. DOI : 10.3917/i2d.172.0067. URL : https://www.cairn.info/revue-i2d-information-donnees-et-documents2017-2-page-67.htm

41. Verdoni, N. (2014). Gérer un réseau de commerce organisé: Approche stratégique et techniques d'animation opérationnelle, l'œil du cyclone éditions, $58 \mathrm{p}$.

42. Wanlin, P. (2007). «L'analyse de contenu comme méthode d'analyse qualitative d'entretiens : une comparaison entre les traitements manuels et l'utilisation de logiciels » in Recherches qualitatives / HORS SÉRIE / 3, Université du Luxembourg, pp.243-272.

43. Webb, J.W., Tihanyi, L., Ireland, R.D. \& Sirmon, D.G. (2009). " You say illegal, I say legitimate: entrepreneurship in the informal economy" , Academy of Management Review, 34 (3), p. 492-510. 